\title{
Meniskektomie nach traumatischer Ruptur ist nur wenig hilfreich
}

\begin{abstract}
Nach allgemeiner Ansicht haben Patienten mit traumabedingtem Meniskusriss größere Chancen, dass sich ihre Beschwerden durch eine Meniskektomie bessern, als Patienten mit degenerativen Meniskusveränderungen. Das könnte ein Irrtum sein.
\end{abstract}

\begin{abstract}
I den vergangenen Jahren haben mehrere Studien demonstriert, dass eine arthroskopische partielle Meniskektomie gegen die Symptome von arthrotisch verursachten Meniskusrissen nicht mehr ausrichtet als eine Scheinoperation. Anders sei die Situation bei traumatischen Rupturen zu beurteilen - so die bisher herrschende Meinung unter Kniespezialisten. Erkenntnisse einer dänisch-schwedischen Forschergruppe um Jonas Thorlund von der Süddänischen Universität in Odense, weisen allerdings in die entgegengesetzte Richtung.

Die Forscher hatten zwei Gruppen von Patienten miteinander verglichen, die sich nach einer Meniskusruptur einer arthroskopischen Teilentfernung des Meniskus unterzogen. In Gruppe 1 $(\mathrm{n}=246)$ waren die Risse degenerativ bedingt, in Gruppe $2(n=141)$ gingen sie auf eine Verletzung zurück. Dabei wurde über ein Jahr hinweg die Verände-
\end{abstract}

rung der Kniebeschwerden mit dem Knee Injury and Osteoarthritis Outcome Score (KOOS) gemessen, wobei Schmerzen, Symptome, Sport- und Erholungsfunktion sowie Lebensqualität jeweils auf Skalen von 0-100 (bestmögliches Ergebnis) bestimmt wurden. Aus allen vier Skalen wurde der KOOS-Mittelwert bestimmt.

Die Auswertung ergab, dass sich die Lage bei den Patienten mit degenerativ bedingten Meniskusrissen tendenziell deutlicher besserte als bei jenen mit traumatischen Rupturen - im Gegensatz zur landläufigen Annahme. Ausgehend von einem KOOS-Durchschnitt von 45,5 Punkten erreichten die Patienten mit arthrotischen Knien 52 Wochen nach dem Eingriff einen Wert von 66,2. Bei den Trauma-Patienten verlief die Entwicklung von anfangs 46,4 Punkten zu schließlich 61,9 Punkten. Die Differenz zwischen den Gruppen war aber weder statistisch signifikant noch klinisch relevant; für letzteres wurde ein ein Unterschied von 10 KOOS-Punkten definiert.

Rund 48 \% der Patienten mit verletzungsbedingten Rupturen waren nach einem Jahr mit der Kniefunktion nicht zufrieden. Bei jenen mit degenerativ bedingten Rissen sagten dies 37\%. 17\% der Patienten der Traumagruppe und $15 \%$ derjenigen mit Kniearthrose sprachen sogar von einem Therapieversagen.

Fazit: Angesichts ihrer Ergebnisse fordern Thorlund und Kollegen weitere Untersuchungen. Sie verweisen auf die guten Erfahrungen mit konservativer Bewegungstherapie bei Patienten mit degenerativen Meniskus- und Rissen des vorderen Kreuzbandes. Auch die Wirksamkeit der arthroskopischen partiellen Meniskektomie bei traumatischen Rupturen sollte deshalb „in kontrollierten Studien mit Placeboeingriffen und nichtoperativen Behandlungen wie beispielweise Bewegungsübungen“"verglichen werden.

Dr. Robert Bublak

Thorlund JB et al. Patient reported outcomes in patients undergoing arthroscopic partial meniscectomy for traumatic or degenerative meniscal tears: comparative prospective cohort study. BMJ 2017; 356: j356; doi: 10.1136/bmj.j356x

\section{Sport auch für multimorbide Arthrose-Kranke}

\author{
Bewegung ist ein wichtiger Bestandteil der Arthrosetherapie. Bei Patienten, \\ die neben ihrer Kniearthrose noch ein Herz- oder Lungenleiden haben oder \\ zusätzlich an Diabetes erkrankt sind, wird jedoch oft darauf verzichtet. Zu \\ Unrecht, wie eine aktuelle Studie ergab.
}

$\mathrm{N}$ iederländische Rehabilitationsmediziner und Orthopäden hatten 126 Probanden mit Gonarthrose in zwei Gruppen randomisiert. Alle litten zusätzlich an mindestens einer Begleiterkrankung, darunter KHK, Diabetes, COPD oder Adipositas.

Die Patienten der Interventionsgruppe trainierten 20 Wochen lang unter Anleitung eines Physiotherapeuten Kraft und Ausdauer. Die Trainingsintensität wurde anhand der Borg-Skala beurteilt und individuell an den jeweiligen Ge- sundheitszustand adaptiert. Die Patienten der Kontrollgruppe erhielten lediglich ihre gewohnte Medikation.

Die körperliche Leistungsfähigkeit wurde bei allen Probanden zu Studienbeginn sowie nach 10, 20 und schließlich nach 32 Wochen anhand der Subskala "physische Funktion“ des Western Ontario and McMaster Universities Osteoarthritis-Indexes (WOMAC-pf) und des 6-Minuten-Geh-Tests (6-MWT) beurteilt. Die Probanden der Interventionsgruppe schnitten im Studienverlauf sig- nifikant besser ab als die der Kontrollgruppe. Nach drei Monate hatten sich die Teilnehmer der Interventionsgruppe auf der WOMAC-pf-Skala im Schnitt um 11,6 Punkte (33\%) verbessert, im 6-MWT um 59 Meter (15\%). Schwerwiegende trainingsbedingte Zwischenfälle traten nicht auf.

Fazit: Mit einem maßgeschneiderten Trainingsprogramm lassen sich offenbar auch bei Patienten mit Begleiterkrankungen Arthrose-bedingte Beschwerden sicher und effektiv lindern. Dr. Dagmar Kraus

De Rooij M et al. Efficacy of tailored exercise therapy on physical functioning in patients with knee osteoarthritis and comorbidity: a randomized controlled trial. Arthritis Care Res 2017; doi: 10.1002/acr.23013 